\title{
Identidade Dialógica, Alteridade e Afetividade
}

\author{
Sofia Tavares ${ }^{1, *} \&$ Catarina Rosa ${ }^{2}$ \\ ${ }^{1}$ Universidade de Évora, Évora, Portugal \\ ${ }^{2}$ Universidade de Aveiro, Aveiro, Portugal
}

\begin{abstract}
RESUMO - Neste artigo teórico, partimos do princípio de que os processos humanos de significação e de conhecimento se consubstanciam e se desenvolvem em interdependência com os outros, para abordarmos a identidade pessoal como um espaço dialógico entre um Eu e um Outro (uma pessoa, um grupo, uma comunidade, uma sociedade), dinamicamente regulado por afetos. A inerência dialógica da vida psicológica é abordada - i.e., o papel dos outros na constituição da subjetividade humana - e, particularmente, as dimensões afetivas e motivacionais desse processo.
\end{abstract}

PALAVRAS-CHAVE: identidade dialógica, alteridade, afetividade

\section{Dialogical Self, Alterity and Affectivity}

\begin{abstract}
In this theoretical paper we assume that human processes of meaning-making and knowledge are embodied and developed in interdependence with the Other(s) in order to approach personal identity as a dialogical space between the I and the Other (an I-position, a person, a group, a community, a society) dynamically regulated by affection. The dialogical inherence of psychological life is addressed, i.e., the role of otherness in the constitution of human subjectivity and particularly the affective and motivational dimensions of this process.
\end{abstract}

KEYWORDS: dialogical self, alterity, affection

Eu não sou eu nem sou o outro, Sou qualquer coisa de intermédio: Pilar da ponte de tédio

Que vai de mim para o outro. (Mário de Sá-Carneiro, 2000, p. 94)

$\mathrm{Na}$ base de uma abordagem dialógica, encontra-se o pressuposto ontológico de que os seres humanos, e, consequentemente, os seus processos psicológicos, têm uma natureza relacional e comunicacional (Salgado, 2005). Nessa perspetiva, a criação de significado para a existência e experiência humana envolve um jogo relacional, discursivamente sustentado entre um Eu e um Outro (ou múltiplos outros) - presente ou ausente, real ou imaginário'.

Em todo e qualquer ato comunicacional, quem "fala" (faz, pensa ou escreve) dirige-se sempre para algo ou

1 O reconhecimento da importância do Outro na constituição da subjetividade humana rompe com a tradição filosófica moderna, que perspectiva "o 'eu' como uma unidade autoconstituída, independente da existência de um 'outro' e de 'outros', que são singulares e independentes" (Coelho \& Figueiredo, 2003, p. 194) alguém, pelo que qualquer atividade de conhecimento (nomeadamente o autoconhecimento) é uma prática interativa na qual a pessoa se posiciona face ao(s) Outro(s). Equivale ists a afirmar que o desenvolvimento da identidade pessoal é uma realização relacional, ou seja, que só existe no diálogo com os Outros (e com o próprio), sem os quais não se poderá definir (Hermans, 2004). Porém, a noção de diálogo requer não apenas uma orientação para o Outro, mas também uma separação do Outro. Nas palavras de Hermans e Kempen (1993, p. 65): "Alguém diz algo a um outro da sua própria perspetiva”. Assim, Eu e Outro são os dois polos da existência humana e o significado de cada um deriva, precisamente, da coexistência do outro.

Uma das premissas da teoria dialógica da identidade (Hermans \& Hermans-Jansen, 1995; Hermans \& Kempen, 1993; Hermans, Kempen, \& van Loon, 1992) é que quando uma pessoa conta uma história acerca de si mesma ou do mundo, para além da posição particular que ocupa no espaço e no tempo, a partir da qual narra a sua versão dos acontecimentos, dirige-se sempre a outra ou outras

*E-mail: tavares.sofia@uevora.pt

- Submetido: 10/11/2015; Aceito: 02/05/2018. 
posições situadas no seu mundo subjetivo ou no ambiente externo. Isso será o mesmo que dizer que a identidade pessoal - ao invés de uma narrativa acabada que alguém conta a um Outro - é uma história continuamente elaborada e reelaborada por duas ou mais vozes, através de intersecções e trocas dialógicas. Falante e ouvinte são, nessa perspetiva, co-construtores da história.

Eventualmente, todos já tivemos a oportunidade de observar alguém contar diferentes histórias acerca de si ou do mundo ou diferentes versões de uma mesma história, em função da sua audiência. Isto revela que cada unidade da narrativa (i.e., cada significado construído) resulta de um encontro entre vozes: entre a voz do autor e a voz antecipada do destinatário. Assim, quem ouve a história - o Outro influencia o que é dito e como é dito, ou seja, já é parte integrante do autor ou, nas palavras de Hermans (2004), é um outro eu. De igual modo, a pessoa funciona como audiência de si mesma em qualquer construção narrativa. Mesmo quando se dirige a um outro real, a pessoa ouve (lê ou vê) simultaneamente o que está a dizer (a escrever ou fazer), tomando parte de um processo de autodiálogo.

Ao narrar uma história a partir de uma posição particular, para além de um conjunto de significados, a pessoa expressa afetos nos significados comunicados que, por sua vez, traduzem a qualidade do seu envolvimento com a audiência (i.e., outras posições pessoais, pessoas ou objetos a quem a história se dirige) e/ou com o referente (i.e., outras posições pessoais, pessoas ou objetos que fazem parte do conteúdo da história).

Cada voz é estruturalmente constituída por um conjunto diferenciado de cargas afetivas, que se encontram endereçadas para pessoas, acontecimentos, objetos, o próprio ou o seu corpo (Hermans, 2001a; Hermans, Ricks, \& Kempen, 1993). Isto quer dizer que cada significado pessoal encerra um determinado valor, um valor de cariz emocional - e, por essa razão, na terminologia de Hermans, os significados são também designados de valorações.

Desse modo, os afetos, enquanto partes integrantes das valorações, correspondem aos aspectos volitivos da identidade, ou seja, representam as dimensões motivacionais, proativas e intencionais da capacidade auto-organizadora da identidade pessoal nas suas transações com o exterior, expressando uma orientação ou movimento na ação da pessoa no mundo. Daí, Hermans et al. (1993) considerarem o afeto como "o movimento metafórico implícito numa valoração" (p. 219).

A relação entre identidade, alteridade - i.e., a presença do Outro no $\mathrm{Eu}$ - e afetividade foi metodologicamente operacionalizada por Hermans (1987a, 1987b, 1991, 1999) e Hermans e Hermans-Jansen (1995) no método da autoconfrontação, através do qual a pessoa associa um conjunto de valorações (construídas a partir de posições internas e/ou externas significativas da sua identidade pessoal) a um conjunto de afetos. Teoricamente, a articulação desses conceitos - nomeadamente, o papel da afetividade no desenvolvimento de uma identidade dialógica - não tem sido objeto de grande atenção por parte dos teóricos da área. Nesse ponto, pretendemos contribuir para o colmatar dessa lacuna, abordando a inerência dialógica da vida psicológica - i.e., o papel dos outros na constituição da subjetividade humana - e, particularmente, as dimensões afetivas e motivacionais desse processo.

\section{O PRIMADO DA RELAÇÃO E DA ALTERIDADE}

Começamos por recuperar a afirmação de que, no modelo do self dialógico, a subjetividade humana é perspectivada como uma construção relacional entre um Eu e um Outro entre um centro e uma periferia (Holquist, 1990) ou, ainda, entre um ego e um alter (Marková, 2003). Prosseguimos, então, pela decomposição analítica dos conceitos sobre os quais essa subjetividade se constitui.

A pessoa (ou para usarmos a mesma terminologia, o Eu) é, no seio desse modelo, tomada como o ponto de partida de qualquer processo dialógico de construção de significado: é o centro processador da experiência no aqui-e-agora e o agente que ativamente torna a ação no mundo possível. Sem uma construção reflexiva sobre a localização do Eu no momento (em termos de tempo e de espaço) de vida, não seria possível definir qualquer outra posição complementar. Por isso, Valsiner (2007) defende que "qualquer relação dialógica está centrada no eu” (p. 131).

Por outro lado, e nessa perspectiva, o Outro, com quem a pessoa interage dialogicamente na construção de significado, não é apenas tido como um personagem do mundo exterior, mas é, simultaneamente, uma voz que habita o espaço social da pessoa. Quer isso dizer que o Outro, proveniente do "exterior", só adquire existência psicológica quando internalizado pela pessoa como uma posição subjetiva - o que acontece através da experiência de se relacionar intersubjetivamente com ele. Essa dupla localização do Outro no reino objetivo e subjetivo - geradora de tensões e conflitos - cria a alteridade em cada vivência psicológica, através da qual o Outro é sentido como uma experiência pessoal.

Nesse âmbito, Hermans $(2003,2004)$ se refere ao Outro como uma posição externa do repertório de posições da pessoa - ou seja, um outro eu - que, como uma outra pessoa (ou como um objeto personificado), ocupa uma posição no espaço dialógico. Como uma outra posição do Eu, esse Outro tem uma versão dos acontecimentos com um conteúdo e uma estrutura diferente da versão contada a partir da perspetiva atual do Eu (i.e., da posição que o Eu ocupa no aqui e agora).

A consideração do Outro como parte intrínseca do espaço subjetivo (para além da sua eventual existência no mundo externo) implica que este não pode ser abordado apenas como um objeto de relação ou conhecimento. O Outro só 
pode ser conhecido através do diálogo relacionalmente sustentado, no qual lhe é dada a oportunidade de se revelar a partir da sua própria posição (Hermans, 2003). Além disso, pressupõe que o autoconhecimento depende também do que se conhece do Outro (ou, por outras palavras, da relação com esse Outro), que habita o espaço social da identidade pessoal.

Por conseguinte, Hermans (2003) concebe o autoconhecimento como o conhecimento de uma identidade projeto, que vai sendo construída num diálogo contínuo e inacabado com o Outro. Nas suas palavras: "o-selfem-relação-com-o-outro é uma forma de exploração e descoberta social como parte de um diálogo inacabado, tanto externo, com o outro real, como interno, com o outro imaginado" (Hermans, 2003, p. 104). Desse modo, conhecimento e autoconhecimento procedem de forma interrelacionada: o autoconhecimento pressupõe o conhecimento do próprio (i.e., das posições que integram o domínio interno do repertório), mas também do Outro, como um outro eu (i.e., enquanto posição do domínio externo do repertório de posições pessoais) (Hermans, 2003). Assim, "o autoconhecimento é, então, a descoberta da alteridade do outro e, como consequência, o meu autoconhecimento aumenta quando a alteridade do outro é admitida e explorada como parte do domínio externo do meu self' (Hermans, 2003, p. 104).

Em conformidade, é possível, agora, afirmar que todos os processos humanos e todas as funções psicológicas têm a sua gênese nas relações sociais com os outros (significativos e culturalmente generalizados). As pessoas se desenvolvem de forma interdependente, ou seja, só existem em si e para si, quando e pelo fato de existirem para outros. Sem o reconhecimento dos outros, "a nossa subjetividade, a nossa autonomia, a nossa liberdade autoconsciente não têm sentido" (Auerbach \& Blatt, 2001, p. 430).

Um exemplo paradoxal do quanto a nossa existência se encontra dependente de um Outro independente é o de que mesmo quando alguém pretende afirmar a sua independência, essa depende do seu reconhecimento pelos outros. Assim, o Eu emerge por referência a um Outro: não há subjetividade sem intersubjetividade, não há $\mathrm{Eu}$ sem Outro (Auerbach \& Blatt, 2001; Coelho \& Figueiredo, 2003; Góes, 2000; Salgado \& Hermans, 2005; Valsiner, 1994). São as relações de partilha entre diferentes subjetividades que conduzem à emergência de diferenciação, no mundo subjetivo, dos processos psicológicos individuais.

É um fato que, desde o início da vida, as relações interpessoais são parte integrante do Eu e os Outros significativos personagens das autonarrativas. Como descreve Bertau (2004), desde muito cedo os seres humanos se relacionam com o mundo, respondendo de forma contingente às estimulações sociais por meio de gestos difusos e indiferenciados (e.g., sorrindo perante o sorriso dos outros, chorando perante o choro dos outros, experienciando as propriedades internas dos gestos e expressões que percebem nos gestos e expressões exibidos pelos outros).
Progressivamente, começam a perceber os outros como tendo uma existência separada da sua, um corpo distinto do seu. Os atos sociais difusos e as rotinas ritmadas entre crianças e adultos evoluem, então, para trocas mútuas entre parceiros que se entendem como intencionais e se posicionam perante uma certa perspetiva do outro e do mundo. A precoce distinção entre $e u$ e $t u$ e a capacidade para assumir a perspectiva do outro marcam o início de um processo de desdobramento pessoal, em que surgem novas vozes e se multiplicam as possibilidades de diálogo entre diferentes posições (Bertau, 2004). Daqui se depreende que a alteridade é uma experiência virtualmente onipresente na existência humana.

Da perspectiva dialógica, a subjetividade humana compreende um $\mathrm{Eu}$ e um Outro - enquanto elementos distintos, mas funcionalmente interdependentes de um mesmo sistema - e emerge da tensão dinâmica entre os dois: a necessidade de relacionamento e a necessidade de diferenciação (Auerbach \& Blatt, 2001). Os seres humanos são seres relacionais nos aspectos sociais e comunicacionais da sua existência, mas têm, igualmente, existências independentes - princípio da "separação inclusiva" de Valsiner (2007). A separação dos parceiros de uma comunicação é, desse ponto de vista, um aspecto crucial para que esses possam manter um diálogo significativo e sensível à perspetiva do outro, ou seja, para que sejam seres verdadeiramente sociais (Bertau, 2004).

Para o dialogismo, nenhuma forma de vida humana é possível senão através do relacionamento dialógico com o Outro e com o mundo. Porém, a noção de diálogo implica não apenas uma orientação para o Outro, mas também uma separação do Outro. Qualquer interação dialógica envolve dois movimentos complementares: um, em que o Eu se dirige para o Outro, num movimento centrífugo de entrar em contacto com o espaço subjetivo do Outro de forma a reconhecer e a assumir a sua perspetiva; e um movimento centrípeto de centração em si, na medida em que a interpretação desse distinto ponto de vista e a resposta ao mesmo se fazem sempre através dos termos do próprio $\mathrm{Eu}$ - o que requer simultaneamente uma separação do Outro (Hermans \& Kempen, 1993). Se a pessoa assumisse por completo a perspectiva do Outro, deixaria de ser capaz de refletir por si e sobre si, mas, por outro lado, caso se afastasse totalmente da posição do Outro, nunca seria capaz de compreender a sua perspectiva (Valsiner, 2007).

É do paradoxo de sermos simultaneamente semelhantes e diferentes dos outros que resulta o diálogo inovador. As comunicações (tanto com os outros, como com o próprio) só são significativas, em termos de progresso, na forma de pensar ou sentir dos seus participantes, se essas, para além de serem viáveis - pela partilha de algum patrimônio linguístico e cultural comum -, envolverem posições diferentes. Caso contrário, se os participantes tiverem a mesma perspectiva do mundo e partilharem a mesma informação, não será de esperar muito progresso dessa negociação (Hermans, 2004). 
No final, Eu e Outro permanecerão como perspectivas distintas, irredutíveis e assimétricas, capazes apenas de se encontrarem no espaço e no tempo da autonarrativa. Sem a perspetiva do Outro, nunca nos conheceremos de fato, mas, por outro lado, nunca teremos um conhecimento completo da perspectiva desse Outro (Barresi, 2002; Hermans, 2001a, 2003, 2004). Assim, o Eu nunca poderá se fechar ao exterior ou identificar-se completamente com o Outro. Como sugere Barresi (2002, p. 249) "mesmo que o self pudesse juntar o seu próprio passado e futuro no presente, esse nunca poderia incluir a perspectiva ou atividade do outro. O outro é sempre um permanente desconhecido". Por essa razão, a tensão entre o Eu e o Outro nunca se consuma e a autonarrativa nunca tem um final unificador.

\section{AFETIVIDADE E MOTIVAÇÃO}

Nas suas interações dialógicas, o Eu e o Outro não comunicam apenas significados, mas, juntamente com esses, afetos e sentimentos ${ }^{2}$. Segundo Stiles, Osatuke, Glick e Mckay (2004), qualquer encontro com o Outro evoca uma reação afetiva (ou avaliativa) no sujeito que reflete a valência positiva ou negativa do acontecimento relacional. Pelo que "sentir é relacionar-se" (Salgado, 2006).

Comungando dessa mesma perspetiva, mas partindo de uma abordagem semiótica, Valsiner (2007) afirma que "a vida psicológica humana, nas suas formas mediada por signos, é afetiva na sua natureza" (p. 265). Do seu ponto de vista, os significados não são mais do que instrumentos semióticos que permitem organizar os afetos emergentes nas interações da pessoa com o mundo e com os outros. $\mathrm{Ou}$ seja, as pessoas atribuem significado ao fluxo constante de experiências através dos afetos que, por sua vez, encontramse culturalmente organizados pelo uso e criação de signos. Assim, todos os encontros com o mundo envolvem afetos e, eventualmente, interpretações sobre esses afetos - i.e., sentimentos.

Mikael Leiman (2002) partilha da noção de que o sentimento é um fenômeno semiótico que une a pessoa aos que a rodeiam. Leiman (2004) postula que em cada elocução o autor expressa uma posição (i.e., uma atitude, uma avaliação) perante o destinatário (voz interna ou entidade do mundo à qual a elocução é dirigida) e o referente (voz interna ou entidade do mundo acerca da qual a elocução é construída) da mesma. Partindo dessa leitura, Stiles et al (2004) sugerem que o conceito de posição se aproxima do de expressão emocional. Do seu ponto de vista, a expressão emocional é "uma (forte) posição [que] o autor toma relativamente à expressão do destinatário ou [do] seu referente" (Stiles et al., 2004, p. 98).

Assumir uma posição é, então, ocupar uma estância específica perante o Outro, da qual se avalia, simultaneamente, o Outro e o mundo ao redor. Cada voz (i.e., cada gesto,

2 Stiles et al. (2004) distinguem, com base em António Damásio, diferentes níveis no processo emocional, utilizando, para esse propósito, os termos afeto e sentimento. De acordo com esses autores, o afeto (emoção, em Damásio) corresponde a uma resposta automática e muito rápida do organismo perante novas experiências. Enquanto, por sua vez, o sentimento corresponde ao reconhecimento do afeto (i.e., mapear as alterações no estado do organismo associadas ao evento ativador). O sentimento é um processo mais lento e duradouro, através do qual o centro atual processador da experiência (i.e., a voz que detém a palavra no momento) valora a experiência. elocução ou ação) é estruturalmente constituída por um conjunto diferenciado de cargas afetivas, que se encontram endereçadas ao(s) seu(s) destinatário(s) e/ou referente(s) (Hermans, 2001a; Hermans et al., 1993). Assim, todos os atos comunicacionais veiculam não apenas significados, mas também, intrinsecamente, elementos avaliativos de natureza emocional $^{3}$ - transformando o espaço relacional num campo de valores energético (Salgado, 2005, 2006).

$\mathrm{O}$ valor positivo ou negativo que um determinado encontro adquire para a pessoa não surge por acaso, antes reflete o grau de gratificação do motivo que rege, naquele instante, a atividade do sujeito e as suas transações com o exterior. Os afetos são os representantes das dimensões motivacionais e intencionais que organizam as permutas das pessoas com o exterior e expressam a orientação da ação imediata da pessoa no mundo. E, nesse sentido, são "o combustível e o guia (e outras vezes a âncora) do self motivado, direcionando a cognição para resolver o que interessa ao self" (Whelton \& Greenberg, 2004, p. 112).

Por regularem as relações da pessoa consigo, com os outros e com o mundo, os afetos e os sentimentos são considerados os componentes dinâmicos da pessoa-emrelação (Salgado, 2005, 2006). Porém, enquanto "estados relacionais" (Trevarthen, as cited in Salgado, 2006), os sentimentos não só têm potencial para aproximar os parceiros de uma comunicação, como também para os afastar. Isto quer dizer que os sentimentos refletem não apenas concórdia e coordenação, mas também tensão, oposição e conflito. Assim, sentimentos agradáveis (tais como: alegria, prazer, felicidade e tranquilidade) encontram-se presentes em momentos de sintonia com o Outro, transmitindo a sensação de gratificação e de entendimento perfeito, enquanto que, por outro lado, sentimentos desprazerosos (tais como preocupação, infelicidade, desânimo e desapontamento) assinalam encontros em que há perda ou oposição entre o Eu e o Outro e em que as intenções são frustradas (Salgado, 2005).

Nas relações humanas - e partindo do pressuposto de que os afetos se encontram sempre dirigidos para (ou sobre) alguém -, para além de ser possível identificar a

3 A teoria da valoração de Hermans assenta precisamente no pressuposto de que todos os significados pessoais têm uma componente afetiva ou avaliativa, o que explicará, provavelmente, que, na sua terminologia, os significados sejam designados como "valorações". 
presença de afetos positivos e negativos, é também possível distinguir afetos dirigidos para o próprio (através dos quais o Eu é experienciado como uma entidade autônoma e autocompetente, dos quais são exemplos: os afetos de autoestima, força, autoconfiança e orgulho) de afetos endereçados para os Outros (sinalizadores de experiências de cooperação e de reciprocidade com os outros ou de proximidade a algo ou alguém, como são exemplos os afetos de carinho, amor, ternura e intimidade) (Hermans \& Hermans-Jansen, 1995).

Por sua vez, essas duas categorias afetivas caracterizam a tendência subjacente nas interações humanas: os afetos dirigidos para o próprio assinalam relações em que o $\mathrm{Eu}$ procura manter ou aumentar a sua separação, autonomia e independência face ao Outro e ao mundo (i.e., movimento centrípeto); e quando, por outro lado, o Eu procura interdependência e abertura ao mundo e aos outros (i.e., o movimento centrífugo), os afetos tendem a ser dirigidos mais para o Outro do que para o próprio. De acordo com Hermans e Kempen (1993), apesar de esses dois movimentos serem simultâneos, em determinados momentos um pode dominar sobre o outro (cf. Tavares, Salgado, \& Gonçalves, 2006).
Dentro dessa perspetiva, esses dois tipos de posicionamento - característicos das interações humanas - podem ser conceitualizados como dois sistemas motivacionais distintos (ainda que complementares). Seguindo a proposta de Hermans e Hermans-Jansen (1995), em toda a atividade de construção de significado podem distinguir-se duas orientações ou motivações complementares: o motivo de autovalorização ou motivo $\mathrm{S}$ (que se refere à procura de reconhecimento, sucesso $\mathrm{e}$ poder) e o motivo de contato e de união com os outros ou motivo $\mathrm{O}$ (que se refere às necessidades de amor, intimidade e carinho com o outro).

Quando as forças centrípetas dominam sobre as centrífugas e o sujeito se dirige afetivamente para si, as preocupações pessoais surgem como o motivo principal, na situação inversa, as preocupações com os outros tornam-se preponderantes. Essas duas orientações ou motivações não são mutuamente exclusivas, mas uma pode dominar sobre a outra. Diferentes acontecimentos e interações podem ativar os dois motivos ou ativar mais um do que outro, sendo que os afetos resultam da satisfação -ou não- desses motivos (Hermans \& Hermans-Jansen, 1995).

\section{CONCLUSÃO}

A natureza relacional da existência humana tem, como principal característica, o endereçamento (i.e., o que é dito é sempre endereçado a alguém). Dirigir-se a alguém é uma particularidade constitutiva da existência humana, sem a qual não há (nem poderia haver) vida. Tudo o que fazemos ou pensamos e dizemos ou escrevemos, fazemo-lo ou pensamo-lo e dizemo-lo ou escrevemo-lo para uma outra pessoa, presente ou ausente (Salgado \& Gonçalves, 2007). $\mathrm{O}$ endereçamento comunicacional ao Outro tem sempre dois lados: o da aproximação ao Outro, das comunalidades e da identificação e o da separação, da independência, das diferenças e da autodefinição (Auerbach \& Blatt, 2001; Hermans, 2001b; Linell, 2005).
O posicionamento dinâmico da pessoa entre esses dois polos, presentes em todas as interações humanas, é regulado por afetos. Ou seja, em qualquer relação de construção de significado as pessoas aproximam-se e/ou afastam-se, em função do que sentem e que, por sua vez, encontra correspondência na satisfação ou não das necessidades que orientam, naquele momento, a sua ação no mundo. Quando os afetos dominantes se voltam para os outros e para as necessidades de aprovação e de aceitação, por parte daqueles com quem se relaciona, a pessoa move-se no sentido da comunhão e da identificação com o Outro e, por outro lado, quando prevalecem as autopreocupações em torno da eficácia e da competência pessoal, os afetos centram-se no próprio, que assim se afasta mais do Outro.

\section{REFERÊNCIAS}

Auerbach, J. S., \& Blatt, S. J. (2001). Self-reflectivity, intersubjectivity, and therapeutic change. Psychoanalytic Psychology, 18(3), 427-450.

Barresi, J. (2002). From "the thought is the thinker" to "the voice is the speaker": William James and the dialogical self. Theory \& Psychology, 12(2), 237-250.

Bertau, M.C. (2004). Developmental origins of the dialogical self: Some significant moments. In H.J.M. Hermans \& G. Dimaggio (Eds.), The dialogical self in psychotherapy (pp. 29-42). New York: Brunner \& Routledge.

Coelho, N. E., \& Figueiredo, C. (2003). Patterns of intersubjectivity in the constitution of subjectivity: Dimensions of otherness. Culture \& Psychology, 9(3), 193-208.

Góes, M. C. (2000). The microgenetic analysis in the historiccultural approach: A perspective for the study of the constitution of subjectivity. Cadernos Cedes, 20(50), 9-25.
Hermans, H. J. M. (1987a). Self as organized system of valuations: Toward a dialogue with person. Journal of Counseling Psychology, 34(1), 10-19.

Hermans, H. J. M. (1987b). The dream in the process of valuation: A method of interpretation. Journal of Personality and Social Psychology, 53(1), 163-175.

Hermans, H. J. M. (1991). The person as co-investigator in selfresearch: Valuation theory. European Journal of Personality, 5(3), 217-234.

Hermans, H. J. M. (1999). Self-narratives as meaning construction: The dynamics of self-investigation. Journal of Clinical Psychology, 55(10), 1193-1211.

Hermans, H. J. M. (2001a). The construction of a personal position repertoire: Method and Practice. Culture \& Psychology, 7(3), 323-366.

Hermans, H. J. M. (2001b). The dialogical self: Toward a theory of personal and culture positioning. Culture \& Psychology, 7(3), 243-281. 
Hermans, H. J. M. (2003). The construction and reconstruction of dialogical self. Journal of Constructivist Psychology, 16, 89-130.

Hermans, H. J. M. (2004). The dialogical self: between exchange and power. In H. J. M. Hermans \& G. Dimaggio (Eds.), The dialogical self in psychotherapy (pp. 13-28). New York: Brunner-Routledge.

Hermans, H. J. M., \& Hermans-Jansen, E. (1995). Self-narratives: The construction of meaning in psychotherapy. New York: The Guilford Press.

Hermans, H. J. M., \& Kempen, H. J. H. (1993). The dialogical self: Meaning as movement. California: Academic Press.

Hermans, H. J. M., Kempen, H. J. G., \& van Loon, R. J. P. (1992). The dialogical self: Beyond individualism and rationalism. American Psychologist, 47(1), 23-33.

Hermans, H. J. M., Ricks, T. I., \& Kempen, H. J. G. (1993). Imaginal dialogues in the self: Theory and method. Journal of Personality, 61(2), 207-236.

Holquist, M. (1990). Dialogism: Bakhtin and his world. London: Routledge.

Leiman, M. (2002). Toward semiotic dialogism: The role of sign mediation in the dialogical self. Theory \& Psychology, 12(2), 221-235.

Leiman, M. (2004). Dialogical sequence analysis. In H. J. M. Hermans \& G. Dimaggio (Eds.), The dialogical self in psychotherapy (pp. 255-269). New York: Brunner-Routledge.

Linell, P. (2005). Essentials of Dialogism: Aspects and elements of a dialogical approach to language, communication and cognition (Manuscrito não publicado). Recuperado de http://www.liu.se/ isk/research/per_li/linell_essentials-of-dialogism_050625.pdf.

Marková, I. (2003). Constitution of the self: Intersubjectivity and dialogicality. Culture and Psychology, 9(3), 249-259.

Sá-Carneiro, M. (2000). Poesias ( $1^{\mathrm{a}}$ ed.). Lisboa: Editora Ulisseia.

Salgado, J. (2005, September). Between you and I: Affectivity and motivation in a dialogical self. Paper presented at the symposium Self Development from a Sociocultural Perspective, ISCAR, Sevilla, Spain.
Salgado, J. (2006). The feeling of a dialogical self: Affectivity, agency and otherness. In L. M. Simão \& J. Valsiner (Eds.), For otherness in question: Labyrinths of the self (pp. 53-71). Carlotte, North Caroline: IAP.

Salgado, J., \& Gonçalves, M. (2007). The dialogical self: Social, personal and (un)conscious. In A. Rosa \& J. Valsiner (Eds.), The Cambridge handbook of socio-cultural psychology. Cambridge, UK: Cambridge University Press.

Salgado, J., \& Hermans, H. J. M. (2005). The return of subjectivity: From a multiplicity of selves to the dialogical self. ElectronicJournal of Applied Psychology, 1, 3-13. Disponível em: http:// www.swin.edu.au/lib/r/onlinejournals/ejap/

Stiles, W. B., Osatuke, K., Glick, M. J., \& Mackay, H. C. (2004). Encounters between internal voices generate emotion: An elaboration of the assimilation model. In H.H. Hermans \& G. Dimaggio (Eds.), The dialogical self in psychotherapy (pp. 91-107). New York: Brunner-Routledge.

Tavares, S., Salgado, J., \& Gonçalves, M. (2006). The psychotherapist's social role under a dialogical perspective: A study of the personal construction of "I as psychotherapist". European Journal of School Psychology, 4(2), 349-372.

Trevarthen, C. (2001). Intrinsic motives for companionship in understanding: Their origin, development, and significance for infant mental health. Infant Mental Health Journal, 22, 95-131.

Valsiner, J. (1994, March). Subjective construction of intersubjectivity: Semiotic mediation as a process of preadaptation. Paper presented at the conference Social Practices and Symbolic Mediation, Université de Neuchâtel, Neuchâtel, Switzerland.

Valsiner, J. (2007). Culture in minds and societies: Foundations of cultural psychology. London: Sage Publications.

Whelton, W. J. \& Greenberg, L. (2004). From discord to dialogue: Internal voices and the reorganization of the self in processexperiential therapy. In H. J. M. Hermans \& G. Dimaggio (Eds.), The dialogical self in psychotherapy (pp. 108-123). New York: Brunner-Routledge. 